DOI: $10.17516 / 1997-1397-2020-13-4-387-397$

УДК 512.6

\title{
Centers and Limit Cycles of Generalized Kukles Polynomial Differential Systems: Phase Portraits and Limit Cycles
}

\author{
Ahlam Belfar* \\ Rebiha Benterki ${ }^{\dagger}$ \\ Department of Mathematics \\ Mohamed El Bachir El Ibrahimi University of Bordj Bou Arreridj \\ El Anasser, Algeria
}

Received 06.04.2020, received in revised form 25.05.2020, accepted 16.06.2020

\begin{abstract}
In this work, we give the seven global phase portraits in the Poincaré disc of the Kukles differential system given by

$$
\begin{aligned}
& \dot{x}=-y, \\
& \dot{y}=x+a x^{8}+b x^{4} y^{4}+c y^{8},
\end{aligned}
$$

where $x, y \in \mathbb{R}$ and $a, b, c \in \mathbb{R}$ with $a^{2}+b^{2}+c^{2} \neq 0$.

Moreover, we perturb these system inside all classes of polynomials of eight degrees, then we use the averaging theory up sixth order to study the number of limit cycles which can bifurcate from the origin of coordinates of the Kukles differential system.
\end{abstract}

Keywords: limit cycle, generalized Kukles differential system, averaging method, phase portrait.

Citation: A. Belfar, R. Benterki, Centers and Limit Cycles of Generalized Kukles Polynomial Differential Systems: Phase Portraits and Limit Cycles, J. Sib. Fed. Univ. Math. Phys., 2020, 13(4), 387-397. DOI: 10.17516/1997-1397-2020-13-4-387-397.

\section{Introduction and statement of the main results}

We consider the so-called Kukles homogeneous differential system. Giné [5]

$$
\dot{x}=-y, \quad \dot{y}=x+Q_{n}(x, y),
$$

which has a center at the origin, where $Q_{n}(x, y)$ denotes a homogeneous real polynomial of degree $n$.

In 1999 Volokitin and Ivanov [12] conjectured that systems (1) have a center at the origin definitely if they are symmetric with respect to one of the coordinate axes. For $n=2$ and $n=3$, the authors of the conjecture knew that it holds. Giné [5] in 2002 proved the conjecture for $n=4$ and $n=5$. Giné et al. $[6,7]$ proved the conjecture for all $n$ under an additional assumption, that the authors believe that it is redundant.

The phase portraits for quadratic systems with center written in the form (1), are known, see Vulpe [13]. The phase portraits of cubic differential systems symmetry with respect to a straight line are also known and in particular those of system (1) with $n=3$, see Buzzi et al. [3], see also Malkin [11]; Vulpe Sibirskii [13] and Żołądek [14,15]. The phase portraits of systems (1) with $n=4$ follows from Benterki and Llibre [1]. Llibre and Silva [9, 10] classified the phase portraits of the systems (1) for $n=5,6$. The phase portraits of systems (1) with $n=7$ follows from Benterki and Llibre [2].

\footnotetext{
*Ahlam.belfar@univ-bba.dz

${ }^{\dagger}$ r.benterki@univ-bba.dz

(c) Siberian Federal University. All rights reserved
} 
In our work, we classify the global phase portraits of the polynomial differential system

$$
\begin{aligned}
& \dot{x}=-y, \\
& \dot{y}=x+a x^{8}+b x^{4} y^{4}+c y^{8} .
\end{aligned}
$$

The first main objective of this work is to study the phase portraits in the Poincaré disc of the differential system (2).

The second objectif, is to give the number of limit cycles which can bifurcate from the origin of coordinates of system (2) when we perturb them inside all classes of polynomial of eight degree, and we do this by using the averaging theory up sixth order.

In Section 2 we give more information about the global phase portraits of the polynomial differential system (2).

Our first main result is given in the following Theorem.

Theorem 1. The set of all global phase portraits in the Poincaré disc of the differential system (2) with $a^{2}+b^{2}+c^{2} \neq 0$, are topologically equivalent to the phase portraits given in Fig. 1.

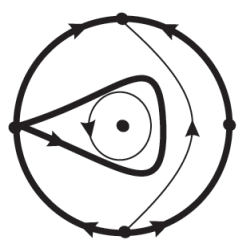

1. $S=10, R=2$

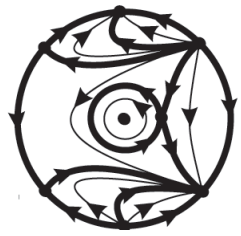

4. $S=22, R=7$

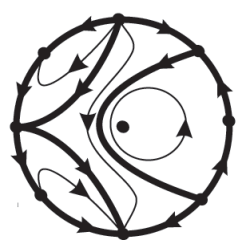

2. $S=20, R=4$

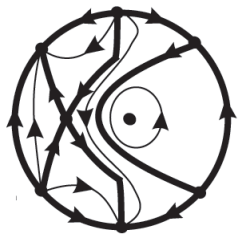

5. $S=19, R=5$

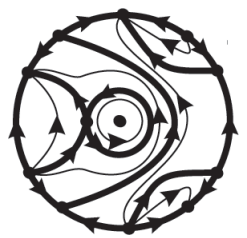

7. $S=29, R=6$

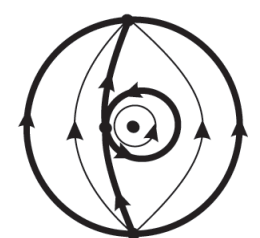

3. $S=9, R=3$

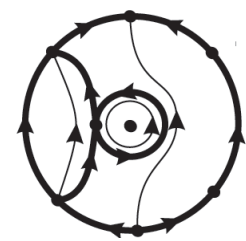

6. $S=18, R=3$

Fig. 1. Global phase portraits of differential system (2)

Theorem 1 is proved in Section 3.

When we perturbed the polynomial differential system (2) with polynomials of degree eight, we get

$$
\begin{aligned}
& \dot{x}=-y+\sum_{s=1}^{6} \varepsilon^{s} \sum_{0 \leqslant i+j \leqslant 8} \alpha_{i j}^{(s)} x^{i} y^{j}, \\
& \dot{y}=x+a x^{8}+b x^{4} y^{4}+c y^{8}+\sum_{s=1}^{6} \varepsilon^{s} \sum_{0 \leqslant i+j \leqslant 8} \beta_{i j}^{(s)} x^{i} y^{j},
\end{aligned}
$$

where $i, j \in \mathbb{N}$. For more information about the averaging theory of higher order see Section 5 .

Our second main result is given in the following theorem. 
Theorem 2. The number of limit cycles of the differential system (3) with $\varepsilon \neq 0$ is

(a) 0 if we use the averaging theory of order 1 or 2,

(b) 1 if we use the averaging theory of order 3 or 4,

(c) 2 if we use the averaging theory of order 5 or 6 .

We give the Proof of Theorem 2 in Section 5.

\section{Preliminaries}

In this section, we give some basic results which are necessary to study the behavior of the trajectories of a planar differential systems near infinity. Let $X(x, y)=(P(x, y), Q(x, y))$ represent a vector field to each system which we are going to study its phase portraits, then for doing this we use the so called a Poincaré compactification. We consider the Poincaré sphere $\mathbb{S}^{2}=\left\{(x, y, z) \in \mathbb{R}^{3}: x^{2}+y^{2}+z^{2}=1\right\}$, and we define the central projection $f: T_{(0,0,1)} \mathbb{S}^{2} \longrightarrow \mathbb{S}^{2}$ (with $T_{(0,0,1)} \mathbb{S}^{2}$ the tangent space of $\mathbb{S}^{2}$ at the point $(0,0,1)$ ), such that for each point $q \in T_{(0,0,1)} \mathbb{S}^{2}, T_{(0,0,1)} \mathbb{S}^{2}(q)$ associaltes the two intersection points of the straight line which connects the point $q$ and $(0,0))$. The equator $\mathbb{S}^{1}=\left\{(x, y, z) \in \mathbb{S}^{2}: z=0\right\}$ represent the infinity points of $\mathbb{R}^{2}$. In summary we get a vector field $\mathcal{X}^{\prime}$ defined in $\mathbb{S}^{2} \backslash \mathbb{S}^{1}$, which is formed by to symmetric copies of $\mathcal{X}$, and we prolong it to a vector field $p(X)$ on $\mathbb{S}^{2}$. By studiying the dynamics of $p(X)$ near $\mathbb{S}^{1}$ we get the dynamics of $\mathcal{X}$ at infinity. We need to do the calculations on the Poincaré sphere near the local charts $U_{i}=\left\{Y \in \mathbb{S}^{2}: y_{i}>0\right\}$, and $V_{i}=\left\{Y \in \mathbb{S}^{2}: y_{i}<0\right\}$ for $i=1,2,3$; with the associated diffeomorphisms $F_{i}: U_{i} \longrightarrow \mathbb{R}^{2}$ and $G_{i}: V_{i} \longrightarrow \mathbb{R}^{2}$ for $i=1,2,3$. After a rescaling in the independent variable in the local chart $\left(U_{1}, F_{1}\right)$ the expression for $p(X)$ is

$$
\dot{u}=v^{n}\left[-u P\left(\frac{1}{v}, \frac{u}{v}\right)+Q\left(\frac{1}{v}, \frac{u}{v}\right)\right], \quad \dot{v}=-v^{n+1} P\left(\frac{1}{v}, \frac{u}{v}\right) ;
$$

in the local chart $\left(U_{2}, F_{2}\right)$ the expression for $p(X)$ is

$$
\dot{u}=v^{n}\left[P\left(\frac{u}{v}, \frac{1}{v}\right)-u Q\left(\frac{u}{v}, \frac{1}{v}\right)\right], \quad \dot{v}=-v^{n+1} Q\left(\frac{u}{v}, \frac{1}{v}\right) ;
$$

and for the local chart $\left(U_{3}, F_{3}\right)$ the expression for $p(X)$ is

$$
\dot{u}=P(u, v), \quad \dot{v}=Q(u, v) .
$$

\section{Study of phase portraits}

In what follows we shall study the phase portraits of the polynomial differential system (2) with $(a, b, c) \neq(0,0,0)$.

Remark 3. System (2) is invariant under the change $(t, x, y) \rightarrow(-t,-x, y)$. Hence, the phase portrait of system (2) is symmetric with respect to the $x$-axis.

Remark 4. System (2) is also invariant under the change

$$
(x, y, t, a, b, c) \rightarrow(-x, y,-t,-a,-b,-c)
$$

then we only need to study the phase portrait of system (2) when $(a=0, b \geqslant 0$ and $c \geqslant 0)$, $(a=0, b>0$ and $c<0),(a>0, b \geqslant 0$ and $c \geqslant 0),(a<0, b>0$ and $c=0),(a>0, b \geqslant 0$ and $c<0),\left(a>0, b^{2}-4 a c=0\right.$, and $\left.b<0\right)$ and $\left(a>0, b^{2}-4 a c>0, b<0\right.$ and $\left.c>0\right)$. 


\subsection{Configurations of singular points}

To study the phase portrait of system (2) we identify all the finite singular points and their local phase portrait. We go through the same steps to study the local phase portrait for the infinite ones.

\subsubsection{Finite singular points}

We identify the finite singular points of the generalized kukles polynomial differential system (2) in the following Proposition.

Proposition 5. The differential system (2) has

(i) Two finite singular points, a center at $(0,0)$ and a hyperbolic saddle at $(-\sqrt[7]{1 / a}, 0)$, if $a \neq 0$;

(ii) one singular point at $(0,0)$ wich is a center, if $a=0$.

Proof. Clearly when $a \neq 0$ the system has two equilibria the origin, with eigenvalues $\pm i$, then we take into acount the symmetry of system (2) with respect to $x$-axis, we conclude that the origin is a center. The second equilibria is $(-\sqrt[7]{1 / a}, 0)$ with eigenvalues $\pm \sqrt{7}$. So it is a hyperbolic saddle.

\subsubsection{Infinite singular points}

By using the preliminaries given in Section 2 we study the infinite singular points and their nature in the Poincaré disc.

Proposition 6. In the chart $U_{1}$ system (2) has

(a) The origin as a linearly zero infinite singular point, and its local phase portrait consists of four hyperbolic sectors, if $a=0, b \geqslant 0$ and $c \geqslant 0$;

(b) three infinite singular points, the origin mentioned in the previous case and two saddlenodes at $( \pm \sqrt[4]{-b / c}, 0)$, if $a=0, b>0$ and $c<0$;

(c) no singularity, if $a>0, b \geqslant 0$ and $c \geqslant 0$;

(d) two infinite semi-hyperbolic saddle-nodes, $( \pm \sqrt[4]{-a / b}, 0)$, if $c=0, b>0$ and $a<0$;

(e) two infinite semi-hyperbolic saddle-nodes at $\left( \pm \sqrt[4]{\frac{-b-\sqrt{b^{2}-4 a c}}{2 c}}, 0\right)$, if $a>0, b \geqslant 0$ and $c<0$;

(f) two infinite linearly zero singular points $( \pm \sqrt[4]{-2 a / b}, 0)$, such that their local phase portraits consist of two hyperbolic and two parabolic sectors, if $a>0, c>0, b^{2}=4 a c$ and $b<0$;

(g) four infinite semi-hyperbolic saddle-nodes,

$$
\left( \pm \sqrt[4]{\frac{-b+\sqrt{b^{2}-4 a c}}{2 c}}, 0\right), \text { and }\left( \pm \sqrt{\frac{b+\sqrt{b^{2}-4 a c}}{-2 c}}, 0\right)
$$

if $a>0, b^{2}-4 a c>0, b<0$ and $c>0$.

The origin of the chart $U_{2}$ is

(h) a hyperbolic node, which is stable if $c>0$ and unstable if $c<0$; 
(i) a linearly zero singular point, such that its local phase portrait consists of four parabolic sectors, if $c=0$.

Proof. The differential system (2) in the chart $U_{1}$ is given by

$$
\begin{aligned}
& \dot{u}=a+b u^{4}+c u^{8}+v^{7}+u^{2} v^{7}, \\
& \dot{v}=u v^{8} .
\end{aligned}
$$

If $b>0, a=0$ and $c \geqslant 0$ system (4) is written as follows

$$
\begin{aligned}
& \dot{u}=b u^{4}+c u^{8}+v^{7}+u^{2} v^{7}, \\
& \dot{v}=u v^{8} .
\end{aligned}
$$

System (2) has one linealrly zero singular point at the origin. Then to study its local phase portrait we have to do blow-up's. We take the directional blow-up $(u, v) \rightarrow(u, w)$ with $w=v / u$ and by doing the rescaling of the time $u^{3} d t=d s$ we have

$$
\begin{aligned}
& \dot{u}=b u+c u^{5}+u^{4} w^{7}+u^{6} w^{7}, \\
& \dot{w}=-b w-c u^{4} w-u^{3} w^{8},
\end{aligned}
$$

this system has one hyperbolic saddle at $(0,0)$, with eigenvalues $\pm b$. Returning through the change of variables to system (4), we conclude that the local phase portrait at the origin trained by four hyperbolic sectors.

If $a=0, b=0$ and $c>0$, and after taking a rescaling of the time $u^{6} d t=d s$ we get the following system

$$
\begin{aligned}
& \dot{u}=c u^{2}+u w^{7}+u^{3} w^{7}, \\
& \dot{w}=-c u w-w^{8} .
\end{aligned}
$$

System (7) has a linearly zero singular point at the origin. Doing blow- up's by performing the directional $(u, w) \rightarrow(u, z)$ with $z=w / u$ and by doing rescaling of the time $u d t=d s$ we get

$$
\begin{aligned}
& \dot{u}=c u+u^{7} z^{7}+u^{9} z^{7}, \\
& \dot{z}=-2 c z-2 u^{6} z^{8}-u^{8} z^{8} .
\end{aligned}
$$

System (8) has one hyperbolic saddle at the origin with eigenvalues $c$ and $-2 c$. Returning through the change of variables to system (7), we conclude that the local phase portrait at the origin formed by four hyperbolic sectors.

If $b>0, a=0$ and $c=0$ we have the following system

$$
\begin{aligned}
& \dot{u}=b u^{4}+u^{7} w^{7}+u^{9} w^{7}, \\
& \dot{w}=-b u^{3} w-u^{6} w^{8} .
\end{aligned}
$$

Doing a change of variable $u^{3} d t=d s$, we get the following system

$$
\begin{aligned}
& \dot{u}=b u+u^{4} w^{7}+u^{6} w^{7}, \\
& \dot{w}=-b w-u^{3} w^{8} .
\end{aligned}
$$

System (10) has one hyperbolic saddle at the origin with eigenvalues $b$ and $-b$. Returning through the change of variables, we know that the local phase portrait at the origin of system (4), when $a=0, b>0$ and $c=0$, consists of four hyperbolic sectors. Then the statement (a) holds.

If $b>0, a=0$ and $c<0$ system (6) has in addition to the origin (the same case in satement $(a))$ two infinite semi-hyperbolic singular points, namely $( \pm \sqrt[4]{-b / c}, 0)$, with eigenvalues 
$\pm 4 b \sqrt[4]{-b^{3} / c^{3}}$ and 0 . Applying Theorem 2.19 of [4] we know that these points are saddle-nodes. Then statement (b) holds.

If $a>0, b \geqslant 0$ and $c \geqslant 0$ system (4) has no singular point.

If $c=0, b>0$ and $a<0$ system (4) becomes

$$
\begin{aligned}
& \dot{u}=a+b u^{4}+v^{7}+u^{2} v^{7}, \\
& \dot{v}=u v^{8},
\end{aligned}
$$

this system has two semi-hyperbolic singular points, $( \pm \sqrt[4]{-a / b}, 0)$ with eigenvalues $\lambda_{1}=$ $= \pm 4 b\left(-a^{3} / b^{3}\right)^{(1 / 4)}$ and $\lambda_{2}=0$. We perform the translation $u=z \pm(-a / b)^{(1 / 4)}$ to system (11). Applying Theorem 2.19 of [4] we know that the points are saddle-nodes. Then (d) is proved.

If $a>0, c>0, b^{2}=4 a c$, and $b<0$ we get the following system

$$
\begin{aligned}
& \dot{u}=\left(2 a+b u^{4}\right)^{2} /(4 a)+\left(1+u^{2}\right) v^{7}, \\
& \dot{v}=u v^{8} .
\end{aligned}
$$

This system has two singular points $( \pm \sqrt[4]{(-2 a) / b}, 0)$ which are linearly zero. We study at first the point $(\sqrt[4]{(-2 a) / b}, 0)$ after performing the translation $u=z+\sqrt[4]{(-2 a) / b}$. Doing blow-up's by taking the directional $(z, v) \rightarrow(z, w)$ with $w=v / z$, and eleminating the common factor $z$ between $\dot{z}$ and $\dot{w}$, we get the following differential system

$$
\begin{aligned}
\dot{z}= & -8 b z \sqrt{\frac{-2 a}{b}}-24\left(\frac{-2 a}{b}\right)^{\frac{1}{4}} b z^{2}-34 b z^{3}+\frac{1}{a} 14(-2 a / b)^{\frac{3}{4}} b^{2} z^{4} \\
& +\frac{7}{a} \sqrt{\frac{-2 a}{b}} b^{2} z^{5}+\frac{2^{\frac{5}{4}}}{a}\left(\frac{-a}{b}\right)^{\frac{1}{4}} b^{2} z^{6}+w^{7} z^{6}+\sqrt{\frac{-2 a}{b}} w^{7} z^{6} \\
& +w^{7} z^{8}+\frac{b^{2} z^{7}}{4 a}+2^{\frac{5}{4}}\left(\frac{-a}{b}\right)^{\frac{1}{4}} w^{7} z^{7}, \\
\dot{w}= & 8 \sqrt{\frac{-2 a}{b}} b w+24\left(\frac{-2 a}{b}\right)^{\frac{1}{4}} b w z+34 b w z^{2}-\frac{14}{a}\left(\frac{-2 a}{b}\right)^{\frac{3}{4}} b^{2} w z^{3} \\
& -\frac{7}{a} \sqrt{\frac{-2 a}{b}} b^{2} w z^{4}-\frac{2}{a}(-2 a / b)^{\frac{1}{4}} b^{2} w z^{5}-w^{8} z^{5}-\sqrt{\frac{-2 a}{b}} w^{8} z^{5} \\
& -\frac{b^{2} w z^{6}}{4 a}-\left(\frac{-2 a}{b}\right)^{\frac{1}{4}} w^{8} z^{6} .
\end{aligned}
$$

For $z=0$, system (13) has one hyperbolic saddle at the origin with eigenvalues $-8 \sqrt{2} b \sqrt{(-a / b)}$ and $8 \sqrt{2} b \sqrt{(-a / b)}$. Going back through the change of variables to system (12), we conclude that the local phase portrait at the singular point $(\sqrt[4]{(-2 a) / b}, 0)$ formed by two hyperbolic and two parabolic sectors. We get the same local phase portrait for the singular point $(-\sqrt[4]{(-2 a) / b}, 0)$ as the singular point $(\sqrt[4]{(-2 a) / b}, 0)$.

If $a>0, b^{2}-4 a c>0, b<0$ and $c>0$ system (4) has four semi-hyperbolic singular points, $\left( \pm \sqrt[4]{\left(-b+\sqrt{b^{2}-4 a c}\right) / 2 c}, 0\right)$, with eigenvalues $\lambda_{1}=2^{\frac{5}{4}}\left(b^{2}-4 a c\right)^{\frac{1}{4}}\left(\frac{-b-\sqrt{b^{2}-4 a c}}{c}\right)^{\frac{3}{4}}$ and $\lambda_{2}=0$, and the points $\left( \pm \sqrt[4]{\left(-b-\sqrt{b^{2}-4 a c}\right) / 2 c}, 0\right)$, with eigenvalues $\lambda_{1}=-2^{\frac{5}{4}}\left(b^{2}-\right.$ $-4 a c)^{(1 / 4)}\left(\frac{-b-\sqrt{b^{2}-4 a c}}{c}\right)^{\frac{3}{4}}$ and $\lambda_{2}=0$. Hence the four singular points are semi-hyperbolic. After transforming these points to the origin we apply Theorem 2.19 of [4] we know that these points are saddle-nodes. 
In the chart $U_{2}$ the differential system (2) becomes

$$
\begin{aligned}
& \dot{u}=-c u-b u^{3}-v^{3}-a u^{5}-u^{2} v^{3}, \\
& \dot{v}=-c v-b u v-a u^{4} v-b u^{2} v-u v^{4} .
\end{aligned}
$$

If $c \neq 0$ the origin is a hyperbolic node of system (14), with eigenvalues $-c$ and $-c$, then it is stable if $c>0$ and unstable if $c<0$. So statement $(e)$ holds.

If $c=0$, system (14) becomes

$$
\begin{aligned}
& \dot{u}=-b u^{3}-v^{3}-a u^{5}-u^{2} v^{3}, \\
& \dot{v}=-b u v-a u^{4} v-b u^{2} v-u v^{4} .
\end{aligned}
$$

The origin is a linearly zero singular point of the differential system (15). We have to do blowup's to know the local phase portrait at this point. We take the directional blow-up $w=v / u$, and by doing the rescaling $u d t=d s$ and we get the system

$$
\begin{aligned}
& \dot{u}=-b u^{2}-a u^{4}-u^{2} w^{3}-u^{4} w^{3}, \\
& \dot{w}=-b w+u w^{4} .
\end{aligned}
$$

When $u=0$; the origin is the only singular point of system (16), with eigenvalues 0 and $-b$. Then, it is a semi-hyperbolic singular point. By using Theorem 2.19 of [4] we conclude that the origin is a saddle-node. Going back through the change of variables to system (15), we know that its local phase portrait formed by four parabolic sectors.

\section{Phase portraits on the Poincaré disc}

Taking into account the results on the finite and infinite singular points given in Subsections 3.1.1 and 3.1.2, respectively, we shall obtain the different phase portraits of the system (2) that we describe in what follows.

Theorem 7. The phase portraits in the Poincare disc of the two compactified polynomial differential systems $p(\mathcal{X})$ and $p(\mathcal{Y})$ are topologically equivalent if and only if their separatrix configurations $S(p(\mathcal{X})$ ) and $S(p(\mathcal{Y}))$ are topologically equivalent.

Case 1. When $a=0, b \geqslant 0$ and $c \geqslant 0$ system (2) has one finite singular point, a center at $(0,0)$. And from statement $(a)$ of Proposition 6 we know that in the chart $U_{1}$ the system has one singular point at $(0,0)$ wich is linearly zero and its local phase portrait consists of four hyperbolic sectors. From statement $(h)$ of Proposition 6 , the origin of $U_{2}$ is a hyperbolic stable node. So, the phase portrait of system (2) is given by Fig. 1(1), and its immediate that $S=10$ and $R=2$.

Case 2. When $a=0, b>0$ and $c<0$ system (2) has one finite singular point at the origin of coodrinates, wich is a center. From statement $(b)$ of Proposition 6 and in the local chart $U_{1}$ the system has three infinite singular points, a linearly zero singularity at the origin such that its local phase portrait consists of four hyperbolic sectors, and two semi-hyperbolic saddle-nodes. In $U_{2}$ and from statement $(h)$ of Proposition 6 , the origin is a hyperbolic unstable node. Then, the phase portrait in this case is topologically equivalent to Fig. 1(2), and its immediate that $S=20$ and $R=4$.

Case 3. When $a>0, b \geqslant 0$ and $c \geqslant 0$ system (2) has two finite singular points, a center at $(0,0)$ and a hyperbolic saddle at $(-\sqrt[7]{1 / a}, 0)$. From statement $(c)$ of Proposition 6 the system has no singular points in the local chart $U_{1}$. In $U_{2}$ and from statement $(h)$ of Proposition 6 , the origin is a hyperbolic stable node if $c>0$, and from statement $(i)$ of the same proposition the origin is a linearly zero singular point and its local phase portrait formed by four parabolic 
sectors if $c=0$. So, in this case the phase portrait is topologically equivalent to Fig. 1 (3), and its immediate that $S=9$ and $R=3$.

Case 4. When $a<0, b>0$ and $c=0$, this case and the following cases 5, 6 and 7 have the same finite singularities as the case 3 . From statement $(d)$ of Proposition 6 the system has two infinite semi-hyperbolic saddle-nodes in the local chart $U_{1}$. In $U_{2}$ and from statement $(i)$ of Proposition 6 , the origin is a linearly zero singular point with local phase portrait formed by four parabolic sectors. Therefore in this case, the phase portrait of system (2) is topologically equivalent to the Fig. 1 (4), and its immediate that $S=22$ and $R=7$.

Case 5. When $a>0, b \geqslant 0$ and $c<0$ system (2) and from statement (e) of Proposition 6 we obtain that the system has two infinite semi-hyperbolic saddle-nodes in the local chart $U_{1}$. The origin of the chart $U_{2}$ is a hyperbolic unstable node. So, the phase portrait of system (2) is topologically equivalent to Fig. 1(5), and its immediate that $S=19$ and $R=5$.

Case 6. When $a>0, b^{2}=4 a c$ and $b<0$ and from statement $(f)$ of Proposition 6 the system has two infinite linearly zero singular points in the local chart $U_{1}$ and their local phase portraits consist of two hyperbolic and two parabolic sectors. In $U_{2}$ and from statement $(h)$ of Proposition 6, the origin is a hyperbolic unstable node. Then, we conclude that the phase portrait of system (2) is topologically equivalent to Fig. 1(6), and its immediate that $S=18$ and $R=3$.

Case 7. When $a>0, b^{2}-4 a c>0, b<0$ and $c>0$ and from statement $(g)$ of Proposition 6 we obtain that the system has four infinite semi-hyperbolic saddle-nodes in the local chart $U_{1}$. In $U_{2}$ and from statement $(h)$ of Proposition 6, the origin is a hyperbolic unstable node. Therefore in this case, the phase portrait of system (2) is topologically equivalent to the Fig. 1(7), and its immediate that $S=29$ and $R=6$.

To know the number of zeros of a real polynomial, we are going to use the following Theorem.

Descartes Theorem. Consider the real polynomial $p(x)=a_{i_{1}} x^{i_{1}}+a_{i_{2}} x^{i_{2}}+\cdots+a_{i_{r}} x^{i_{r}}$ with $0 \leqslant i_{1}<i_{2}<\cdots<i_{r}$ and $a_{i_{j}} \neq 0$ real constants for $j \in\{1,2, \cdots, r\}$. When $a_{i_{j}} a_{i_{j+1}}<0$, we say that $a_{i_{j}}$ and $a_{i_{j+1}}$ have a variation of sign. If the number of variations of signs is $m$, then $p(x)$ has at most $m$ positive real roots. Moreover, it is always possible to choose the coefficients of $p(x)$ in such a way that $p(x)$ has exactly $r-1$ positive real roots.

\section{Proof of Theorem 2}

Consider system (2), we shall study which periodic solutions of the center become limit cycles when we perturb the center inside the class of polynomial differential systems of degree 8 . This study will be done by applying the averaging theory, we work as follows.

Before doing the scaling $x=\varepsilon X, y=\varepsilon Y$, with $\varepsilon$ is a small parameter we get a new differential system $(\dot{X}, \dot{Y})$. After we perform the polar change of coordinates $X=r \cos \theta, Y=r \sin \theta$, then we get a differential system $(\dot{r}, \dot{\theta})$. We take the independent variable the angle $\theta$ we get the differential equation $d r / d \theta$, and by doing a Taylor expansion up to 6 -th order in $\varepsilon$ we obtain the differential equation

$$
r^{\prime}=\frac{d r}{d \theta}=\sum_{i=0}^{6} \varepsilon^{i} F_{i}(\theta, r)+O\left(\varepsilon^{7}\right) .
$$

The functions $F_{i}(\theta, r) i=1, \ldots, 6$ of the differential system (17) are analytic, and since the independent variable $\theta$ appears through the sinus and cosinus of $\theta$, they are $2 \pi$-periodic. Hence the assumptions for applying the averaging theory given in $[8]$ are satisfied.

To know how the averaging theory for differential equation works we advice the lecture to see [8]. 
We give only the expression of the function $F_{1}(r, \theta)$. The explicit expression of $F_{i}(r, \theta)$ with $i=2, \ldots, 6$ is quite large so we omit them.

The functions $F_{i}(\theta, r) i=1, \ldots, 6$ and $R(t, x, \varepsilon)$ of system (17) are analytic, and since the variable appears through sinus and cosinus of $\theta$, they are $2 \pi$-periodic. Hence the assumptions of Theorem 10 are satisfied.

The expression of $F_{1}(r, \theta)$ is

$$
F_{1}(r, \theta)=+\beta_{00}^{(2)} \sin \theta+\frac{r}{2}\left(\alpha_{10}^{(1)}+\beta_{01}^{(1)}+\left(\alpha_{10}^{(1)}-\beta_{01}^{(1)}\right) \cos 2 \theta+\left(\alpha_{01}^{(1)}+\beta_{10}^{(1)}\right) \sin 2 \theta\right) .
$$

Using the formulas given in section 4.1 of [8] the averaged function of first order is

$$
f_{1}(r)=\left(\alpha_{10}^{(1)}+\beta_{01}^{(1)}\right) r
$$

Clearly equation $f_{1}(r)=0$ has no positive zeros. Thus the averaging method of first order does not provide limit cycles.

We put $\alpha_{10}^{(1)}=-\beta_{01}^{(1)}$ we obtain $f_{1}(r) \equiv 0$. We apply the averaging theory of second order, we get the averaging function of second order.

$$
f_{2}(r)=\left(\alpha_{10}^{(2)}+\beta_{01}^{(2)}\right) r
$$

We see that the equation $f_{2}(r)=0$ has no positive zeros, it follows that there is no limit cycle by applying the averaging theory of second order.

To apply the averaging method of third order we must put $\alpha_{10}^{(2)}=-\beta_{01}^{(2)}$, and we get $f_{2}(r) \equiv 0$. The third averaging function is

$$
\begin{aligned}
f_{3}(r)= & -\left(\beta_{11}^{(1)} \beta_{00}^{(2)}-\beta_{01}^{(3)}+2 \beta_{00}^{(2)} \alpha_{20}^{(1)}-2 \beta_{02}^{(1)} \alpha_{00}^{(2)}-\alpha_{11}^{(1)} \alpha_{00}^{(2)}-\alpha_{10}^{(3)}\right) r \\
& +(1 / 4)\left(3 \beta_{03}^{(1)}+\beta_{21}^{(1)}+3 \alpha_{30}^{(1)}+\alpha_{12}^{(1)}\right) r^{3} .
\end{aligned}
$$

So, $f_{3}(r)$ can have at most one positive real root. Then we have the proof of the theorem for $k=3$.

To apply the averaging method of fourth order, we need to have $f_{3}(r) \equiv 0$, for that we set $\alpha_{10}^{(3)}=\beta_{11}^{(1)} \beta_{00}^{(2)}-\beta_{01}^{(3)}+2 \beta_{00}^{(2)} \alpha_{20}^{(1)}-2 \beta_{02}^{(1)} \alpha_{00}^{(2)}-\alpha_{11}^{(1)} \alpha_{00}^{(2)}$ and $\alpha_{12}^{(1)}=-\left(3 \beta_{03}^{(1)}+\beta_{21}^{(1)}+3 \alpha_{30}^{(1)}\right)$.

The averaging function of fourth order is

$$
f_{4}(r)=r\left(A_{1}+A_{2} r^{2}\right)
$$

where

$$
\begin{aligned}
A_{1}= & \left(\beta_{10}^{(1)} \beta_{11}^{(1)} \beta_{00}^{(2)}+2 \beta_{01}^{(1)} \beta_{02}^{(1)} \beta_{00}^{(2)}-\beta_{00}^{(2)} \beta_{11}^{(2)}-\beta_{11}^{(1)} \beta_{00}^{(3)}+2 \beta_{10}^{(1)} \beta_{00}^{(2)} a_{20}^{(1)}-2 \beta_{00}^{(3)} \alpha_{20}^{(1)}\right. \\
+ & \beta_{01}^{(1)} \beta_{00}^{(2)} \alpha_{11}^{(1)}-\beta_{01}^{(1)} \beta_{11}^{(1)} \alpha_{00}^{(2)}+2 \beta_{02}^{(2)} \alpha_{00}^{(2)}+2 \beta_{02}^{(1)} \alpha_{01}^{(1)} \alpha_{00}^{(2)}-2 \beta_{01}^{(1)} \alpha_{20}^{(1)} \alpha_{00}^{(2)} \\
+ & \left.\alpha_{01}^{(1)} \alpha_{11}^{(1)} \alpha_{00}^{(2)}-2 \beta_{00}^{(2)} \alpha_{20}^{(2)}+\alpha_{00}^{(2)} \alpha_{11}^{(2)}+2 \beta_{02}^{(1)} \alpha_{00}^{(3)}+\alpha_{11}^{(1)} \alpha_{00}^{(3)}+\alpha_{10}^{(4)}+\beta_{01}^{(4)}\right) . \\
A_{2}= & \frac{-1}{4}\left(\beta_{20}^{(1)} \beta_{11}^{(1)}+\beta_{11}^{(1)} \beta_{02}^{(1)}+\beta_{10}^{(1)} \beta_{21}^{(1)}+2 \beta_{01}^{(1)} \beta_{12}^{(1)}-3 \beta_{03}^{(2)}-\beta_{21}^{(2)}+\beta_{21}^{(1)} \alpha_{01}^{(1)}\right. \\
& -\alpha_{20}^{(1)} \alpha_{11}^{(1)}-2 \beta_{02}^{(1)} \alpha_{02}^{(1)}-\alpha_{11}^{(1)} \alpha_{02}^{(1)}+2 \beta_{01}^{(1)} \alpha_{21}^{(1)}+3 \beta_{10}^{(1)} \alpha_{30}^{(1)}+3 \alpha_{01}^{(1)} \alpha_{30}^{(1)} \\
& \left.-3 \alpha_{30}^{(2)}+2 \beta_{20}^{(1)} \alpha_{20}^{(1)}-\alpha_{12}^{(2)}\right) .
\end{aligned}
$$


According to the expression of the function $f_{4}$ we conclude that we can get at most one limit cycle.

Solving $A_{1}=0$ and $A_{2}=0$ we obtain $f_{4}(r) \equiv 0$, so we can apply the averaging theory of order 5 , and its corresponding averaging function is $f_{5}(r)=r\left(B_{1}+B_{2} r^{2}+B_{3} r^{4}\right)$.

The explicit expression of $B_{i}$, with $i=1,2,3$ is quite large so we omit them.

The rank of the largest square matrix of the Jacobian matrix $\mathcal{B}=\left(B_{1}, B_{2}, B_{3}\right)$ is 3 . Then the coefficients $B_{1}, B_{2}$ and $B_{3}$ are linearly independent in their variables. By the Descartes Theorem (or by the roots of a quadratic polynomial in the variable $r^{2}$ ) it follows that we can get at most two positive real roots of $f_{5}(r)$. So statement (c) holds. Solving $B_{1}=0, B_{2}=0$ and $B_{3}=0$ we obtain $f_{5}(r) \equiv 0$.

Now if we apply the averaging method of sixth order we get

$$
f_{6}(r)=\left(K_{1}+K_{2} r^{2}+K_{3} r^{4}\right) r .
$$

In this case and for the same reason as the previous one we will not give the explicit expression of $K_{i}$, with $i=1,2,3$ because it is quite large.

The rank of the Jacobian matrix $\mathcal{K}=\left(K_{1}, K_{2}, K_{3}\right)$ with respect to its variables is 3 . We have three of the coefficients $K_{i}, i=1,2,3$ which are linearly independent in their variables. Therefore by Descartes Theorem, it follows that $f_{6}(r)=0$ can has 2 positive real solutions. Consequently, the differential system (2) has at least 2 limit cycles. This ends the proof of the theorem.

\section{References}

[1] R.Benterki, J.Llibre, Centers and limit cycles of polynomial differential systems of degree 4 via averaging theory, J. Computational and Appl. Math., 313(2017), 273-283.

[2] R.Benterki, J.Llibre, The centers and their cyclicity for a class of polynomial differential systems of degree 7 via averaging theory, J. Computational and Appl. Math., 368(2020), 112456.

[3] C.A.Buzzi, J.Llibre, J.C.Medrado, Phase portraits of reversible linear differential systems with cubic homogeneous polynomial nonlinearities having a non-degenerate center at the origin, Qual. Theory Dyn. Syst., 7(2009), 369-403.

[4] F.Dumortier, J.Llibre, J.C.Artés, Qualitative theory of planar differential systems, Universitext, Spring-Verlag, 2006.

[5] J.Giné, Conditions for the existence of a center for the Kukles homogenenous systems, Comput. Math. Appl., 43(2002), 1261-1269.

[6] J.Giné, J.LLibre, C.Valls, Centers for the Kukles homogeneous systems with odd degree, Bull. London Math. Soc., 47(2015), 315-324.

[7] J.Giné, J.LLibre, C.Valls, Centers for the Kukles homogeneous systems with even degree, J. Appl. Anal. Comp., 7(2017). DOI: [10.11948/2017093]

[8] J.Llibre, D.D.Novaes, M.A.Teixeira, Nonlinearity, 27(2014), 563-583.

DOI: $10.1088 / 0951-7715 / 27 / 3 / 563$

[9] J.Llibre, M.F.da Silva, Int. J. of Bifurcation and Chaos, 26(2016), no. 3, 1650044. DOI: $10.1142 / \mathrm{S} 0218127416500449$

[10] J.Llibre, M.F.da Silva, Global phase portraits of Kukles differential systems with homogenous polynomial nonlinearities of degree 5 having a center, Topological Methods in Nonlinear Analysis, 48(2016), 257-282. 
[11] K.E.Malkin, Criteria for the center for a certain differential equation, Volzhskii Matematicheskii Sbornik, 2(1964), 87-91 (in Russian).

[12] E.P.Volokitin, V.V.Ivanov, Isochronicity and Commutation of polynomial vector fields, Siberian Mathematical Journal, 40(1999), 23-38.

[13] N.I.Vulpe, K.S.Sibirskii, Centro-affine invariant conditions for the existence of a center of a differential system with cubic nonlinearities, Soviet Math. Dokl., 38(1989), 198-201.

[14] H.Żołądek, The classification of reversible cubic systems with center, Topol. Methods Nonlinear Anal., 4(1994), 79-136.

[15] H.Żołądek, Remarks on: The classification of reversible cubic systems with center, Topol. Methods Nonlinear Anal., 8(1996), 335-342.

\title{
Центры и предельные циклы обобщенно-дифференциальных полиномиальных систем Куклеса: фазовые портреты и предельные циклы
}

\author{
Ахлам Белфар \\ Ребиха Бентерки \\ Математический факультет \\ Университет Мохаммеда Эль Бачира Эль Ибрахими \\ Эль Анассер, Алжир
}

Аннотация. В этой работе мы даем семь глобальных фазовых портретов в диске Пуанкаре дифференциальной системы Куклеса, заданной как

$$
\begin{aligned}
& \dot{x}=-y, \\
& \dot{y}=x+a x^{8}+b x^{4} y^{4}+c y^{8},
\end{aligned}
$$

где $x, y \in \mathbb{R}, a, b, c \in \mathbb{R}$ и $a^{2}+b^{2}+c^{2} \neq 0$.

Кроме того, мы возмущаем эту систему внутри всех классов многочленов восьмой степени, а затем используем теорию усреднения до шестой степени для изучения числа предельных циклов, которые могут раздвоиться от начала координат дифференциальной системы Куклеса.

Ключевые слова: предельный цикл, обобщенная дифференциальная система Куклеса, метод усреднения, фазовый портрет. 